\title{
Modification of Species-Based Differential Evolution for Finding All Solutions of Systems of Nonlinear Equations
}

\author{
Said Iskandar ${ }^{1}$, Didi Febrian ${ }^{2}$, Faridawaty Marpaung ${ }^{3}$, Ayu Adela ${ }^{4}$ \\ \{ saidiskandar@unimed.ac.id, febrian.didi@unimed.ac.id, farida2008.unim@gmail.com, \\ ayuadela107@gmail.com\} \\ Department of Mathematics, Faculty of Mathematics and Natural Sciences,State University of \\ Medan ${ }^{1,2,3,4}$
}

\begin{abstract}
Nowadays the root finding problem for nonlinear system equations is still one of the difficult problems in computational sciences. Many attempts using deterministic and meta-heuristic methods have been done with their advantages and disadvantages, but many of them have fail to converge to all possible roots.Differential Evolution (DE), a random search techniques using vector as an alternative solution in the search for finding all ofthe real roots from the system of nonlinear equations. Species-based is one of the methods niching the building sub-populations or species in the area with species-seed functions as its center. This study will describe the modification of existing species-based previously to reduce the computational complexity and run more efficiently. The results of the test function shows species-based modifications can localize all potential roots with one running the program
\end{abstract}

Keywords: Differential Evolution, Species-Based, Nonlinier Equations.

\section{Introduction}

At this time optimization and the root finding problem for nonlinear system equations has an important role in various fields of operational research, industry, finance and management. Optimization problem is a problem of maximizing or minimizing a function of one variable or multiple variables, functions examined included unimodal and multimodal function. In searching for the optimum solution can be done by calculus, numerical and random value (random search). Search the optimum solution by using basic calculus and numerical methods must meet certain requirements. A search solution by using basic calculus method requires an objective function that is continuous or differentiable and has a solution or initial guesses. Numerical methods can not be used directly to search for the optimum solution of an objective function. However the optimum solution can be obtained by finding the roots of the first derivative of the objective function numerically. This means that the use of numerical methods to find the optimum solution also requires the presence of the first derivative of the objective function. Yet in reality often encountered problems with the optimization objective function is discrete and not continuous, not differentiable, has a gradient which fluctuate very quickly, and does not have the guesses/early solution[8].

Nowadays the root finding problem for nonlinear system equations is still one of the difficult problems in computational sciences. the root finding problem a function of one 
variable or multiple variables, functions examined included one or more root in a function. In searching for the root finding problem can be done by calculus, numerical and random value (random search). Search the root solution by using basic calculus and numerical methods must meet certain requirements. A search solution by using basic calculusmethod requires an objective function that is continuous or differentiable and has a solution or initial guesses.

In this discussion we utilize Differential Evolution (DE), the method is to run a random search techniques using vector as an alternative solution in the search for the root solution. DE is one of the evolutionary algorithm has a performance as good as that of other evolutionary algorithms such as genetic algorithm (GA) [2,3,4]. DE was first introduced by Ken Price's and Rainer storn in 1994 [4], excess DE is the evolution experienced by each individual in a population where differentiation and crossovers occur sequentially in each individual randomly selected from the population at any time. Crossover parameters modified to determine the effect on the computing process. Results of the testing showed crossover parameters which are both used in the differential evolution for optimization problems [3]. In one population vector values to which every individual is distributed randomly will turn into a vector value towards the best value that ultimately becomes the maximum and minimum solution to the function. Although it may seem simple DE to localize the global optimum very fast and accurate $[2,8]$.

\section{Researach method}

\subsection{Differential Evolution}

Differential Evolution (DE) is a method developed by Kennethrice and published in October 1994 in the magazine Dr.Dobb's Journal (Priceetal., 2005). This method is a mathematical function of multi dimensional optimization method and included in the group of evolutionary algorithm. The emergence of DE method originates from the business Che by chev polynomial fitting solving problems and generating ideas of using the difference vector to randomize thev ector population. Then along with the development, in ICEO (International Conteston Evolutionary Optimization) first, DE becomes one of the best and genetic algorithm can find the global optimum multi dimensional (ie showing more than one optimum value) with a good probability[3,4].

4]. 


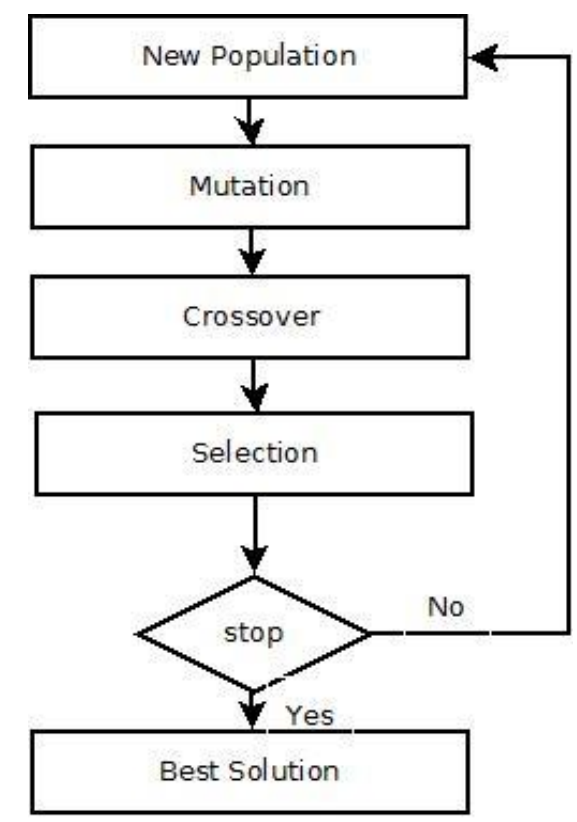

Fig 1. Flowchart for differential evolution

The initial stepofthismethodis theinitializationvectorgeneratedrandomly inareafunctions.

$$
x_{i, j}=x_{i, j_{\min }}+\operatorname{rand}(0,1)\left(x_{i, j_{\max }}-x_{i, j_{\min }}\right)(1)
$$

where $j=1,2, . ., D$ (dimensional vector) and $i=1,2, \ldots, N P$ (the number of vectors in the population), $D$ is the dimension ofa vector, $N P$ is a lot of vectors in a population. Area boundaryfunction of each component of the vector can be written $\min <x_{\mathrm{j}}<\max$, where $j=1,2, \ldots, D$. Onceinitialized, DEwillmutateandme-recombination initial populationtoproducenew populations.

$$
\vec{v}_{i, g}=\vec{x}_{r 0, g}+F\left(\vec{x}_{r 1, g}-\vec{x}_{r 2, g}\right)(2)
$$

where $\vec{v}_{i, g}$ is mutant vector, gisthe vectorgenerationin the process ofmutation. The scale factor, $F \in(0,1)$ is apositivereal numberthatcontrols the developmentofpopulation changesgeneratedbytwo vectorsare randomly selectedfrom the population. $r 0, r 2$ and $r 3$ is the indexnumbertogeneratea randomvectorofthe population. To complete thesearch strategydifferentialmutation, DEalso employs acrossover.

$$
\vec{u}_{i, g}=\left\{\begin{array}{c}
\vec{v}_{i, g} \text { if }(\text { rand }(0,1) \leq C R) \\
\vec{x}_{i} \text { otherwise }
\end{array}\right.
$$

From the equation above, rand $(0,1)$ is a random number. CR is a constant crossover models specified by the manufacturer. If the random value that appears smaller than $\mathrm{CR}$ parameters it will be a new vector will appear from the results of the mutation, otherwise it will use a long vector, then in this optimization process prior to the transfer must meet the 
requirements of crossover parameters. Results of the crossover will be tested with the results of the selection function

\subsection{Species-based differential evolution}

Species-based is one of the methods niching used in multimodal optimization. This method of forming a lot of population in the area function by maintaining the distance (euclidean distance) in the placement of its center point $[2,4,5,6]$. Each population has a radius (r) between the vectors and the population center point. The central point is also called the seed species. Here is the algorithm of formation of species in a population:

1. Spread a random vector in the function area.

2. Sorting each function value of all existing vector, the value of the vector function at its best it is the focal point of the first population.

3. If $r$ is the radius of each population, each located within a radius vector $r$ will be the first population of the population.

4. The rest of the vector that is then in sorting like (step 2). Having obtained the best value performed (step 3). It will get the second population. And so on.

5. The process will stop if there is no more vectors to be in sorting, or all vectors have entered into one of the populations.

6. Calculate the number of vectors in the population, if all ideal minimal amount for poses DE (ideal minimal amount adjusted for optimization functions), if not ideal vector generation is carried out randomly in a radius populsi.

7. All the population will make the process of DE, until every population converges.

8. Stop if it is found that the criteria chill.

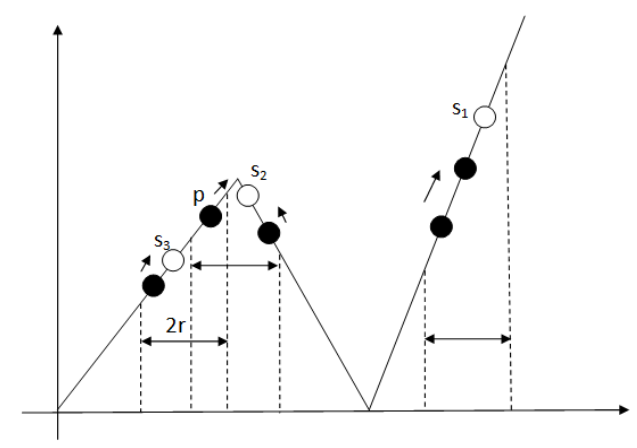

Fig2. Illustrution for Species-Based

In this illustration explained that $s 1, s 2$ and $s 3$ is a vector that is the center of population. Being $\mathrm{p}$ are vectors in the population. The number of vertices in the population can be sure there will be the same as the spread randomly and the proximity of each vector to the center point. Then it could be one of the vectors will get less than ideal vector to make the process of differential evolution[3]

\subsection{Modification species - based differential evolution}

Developmentis donein aspecies-based so thateachpopulationhas astepformationprocesses moreefficient, whileits purpose is: 
1. To avoidsortingrepeatedly toseekseedspeciesin optimization.

2. In ordernumber ofthe vectorin the population hasthe sameidealamountforthedifferential evolution.

3. In order tomore evenlyspread ofseedspeciestoreachall solution of linear function.

Fundamental changesin the algorithm isin the formation ofseedspecies. Furthermore,I call theseedspeciesfeaturedvectorwhichisthe vectorthatwillbe the center ofchange, steps are :

1. Establish afeaturedvectorsfirst. Formationfeaturedvectoristo be raisedfor the first time, in contrast withthe first algorithmthatwas formedfromthe best resultsortingvectorsthat have beengeneratedat random. Vectorseededfirstat the center ofan intervalfunction,

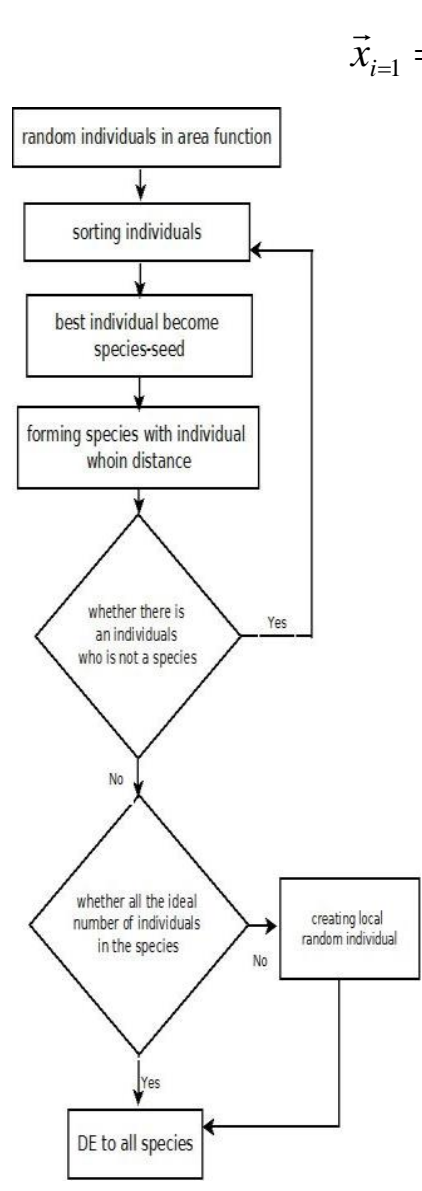

(a)

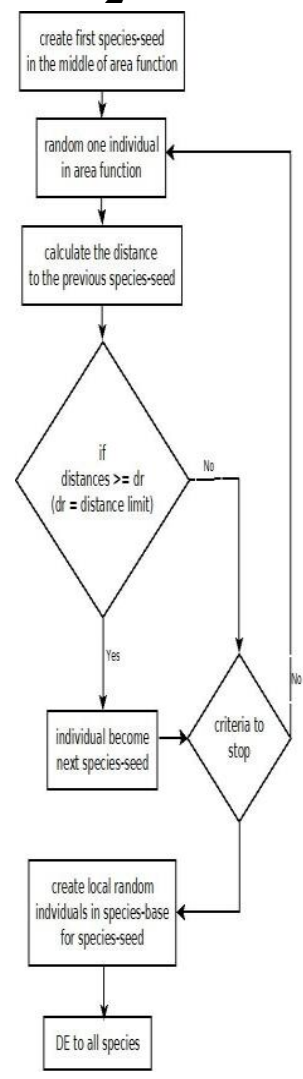

(b)

Fig 3. (a) Species Based Scheme, (b) Modification Species-Based Scheme

2. Establish a featured vectors of the $2 \mathrm{nd}$, $3 \mathrm{rd}$ and so on. In this algorithm we set the minimum distance between vectors featured in accordance with the interests and shape of the optimization function. Vector seeded become a reference for superior vector-2 randomly generated, vector seeded 1 and 2 become a reference for vector- 3 seed. This process continues until This process continues until certain criteria. If the minimum 
distance is not met, then the vector randomly generated vectors not be featured. If we generate 100 random vectors could be a featured vectors which occurred less than 100 .

$$
\begin{aligned}
& \vec{x}_{p}^{*}=\operatorname{rand}(0,1) \cdot\left(x_{j_{\max }}-x_{j_{\min }}\right)+x_{j_{\min }}(5) \\
& p=1,2, \ldots, n \text { (number of trials) } \\
& \operatorname{dist}\left(\vec{x}_{i}, \vec{x}_{p}^{*}\right)=\sqrt{\sum_{i=1}^{N P}\left(x_{i, j}-x_{p, j}^{*}\right)^{2}}(7) \\
& \text { if } \operatorname{dist}\left(\vec{x}_{i}, \vec{x}_{p}^{*}\right) \geq d r \text { then } \vec{x}_{i+1}=\vec{x}_{p}^{*}(8)
\end{aligned}
$$

Where, $d r$ is the amount of distance between vectors $\vec{x}^{*}$ israndomly generated vectors, $i=1,2, \ldots, N P_{\text {seed }}$ (the amount of seed vectors) $j=1,2, \ldots, D$ (dimensionalvector), dististhe distance between two vectors featured,

\section{Result and Discussion}

Considerasystemofnonlinearequationsoftheform

$$
\begin{aligned}
& f_{1}\left(x_{1}, x_{2}, \ldots, x_{n}\right)=0, \\
& f_{2}\left(x_{1}, x_{2}, \ldots, x_{n}\right)=0, \\
& f_{n}\left(x_{1}, x_{2}, \ldots, x_{n}\right)=0,(9)
\end{aligned}
$$

where $\left(x_{1}, x_{2}, \ldots, x_{n}\right) \in D=\left[a 1, b_{1}\right] \times\left[a 2, b_{2}\right] \times \cdots \times\left[a_{n}, b_{n}\right] \mathrm{R}^{n}$ and $f_{i}: D \rightarrow \mathrm{R} \quad i=1,2, \ldots, n$ being continuous functions with atlea stone of themis nonlinear. The above system can be writtenin vector for $\mathbf{f}(\mathbf{x})=0$ where $\mathbf{f}=\left(f_{1}, f_{2}, \ldots, f_{n}\right)^{T}$ and $\mathrm{x}=\left(x_{1}, x_{2}, \ldots, x_{n}\right)^{T}$. Avector $\mathrm{x}^{*}=\left(x^{*}{ }_{1}, x^{*}{ }_{2}, \ldots, x^{*}\right)^{T} \in D$ is called a solution or root of the system if $\mathbf{f}\left(\mathbf{x}^{*}\right)=0$.

There lationship between the optimization problem of a function from $\mathrm{R}^{n}$ to $\mathrm{R}$ and the problem of solving system of nonliner equations is as follows.

$\operatorname{Systemf}(\mathbf{x})=0$ hasasolution $\mathbf{x}=\left(x_{1}, x_{2}, \ldots, x_{n}\right)^{T}$. If the objective function $F$ of the optimization problem, which is defined by

$$
\begin{aligned}
& F(x)=F\left(x_{1}, x_{2}, \ldots, x_{n}\right) \\
& F(x)=\frac{1}{1+\sum_{i=1}^{n}\left|f_{i}\left(x_{1}, x_{2}, \ldots, x_{n}\right)\right|} \\
& F(x)=\frac{1}{1+\sum_{i=1}^{n}\left|f_{i}(x)\right|}
\end{aligned}
$$

Hasthemaximumvalueof1.This would suggest the possible us age of global optimization methods for discovering the solution of nonlinear system Eq.(1). If there exist $\mathbf{x}^{*}$ such that $\left.F(\mathbf{x})^{*}\right)=1$ then $\mathbf{x}^{*}$ is a global optimum of methods for discovering the solution of nonlinear system. If there exist $\mathbf{x}^{*}$ such that $F\left(\mathrm{x}^{*}\right)=1$, then $\mathrm{x}^{*}$ is the root of the system equations. The determination of formula $F(\mathrm{x})$ is motivated by the formula for fitness $F(x)=\frac{1}{1+a b s(f(x))}$ for solving transcendental equations $f(x)=0$ using the genetic algorithm.

Many optimization problems are of highly nonliniear involving many variables under some constraints. Such nonlinearity often result in the multimodal objective function. Hence. Local search algorithm such as hill climbing or steepest descent methods are not suitable. 
Only global search algorithms are suitable for obtaining optimal solutions. Recently several metaheuristic methods have been developed to perform global search.

\subsection{Numerical experiments}

Differential Evolution (DE) is a method developed by Kennethrice and published in October 1994 in the magazine Dr.Dobb's Journal (Priceetal., 2005). This method is a mathematical function of multi dimensional optimization method and included in the group of evolutionary

\subsection{Differential evolution}

Differential Evolution (DE) is a method developed by Kennethrice and published in October 1994 in the magazine Dr.Dobb's Journal (Priceetal., 2005). This method is a mathematical function of multi dimensional optimization method and included in the group of evolutionaryIn order to assess the efficacy of the proposed technique, a set of problems from various benchmark problems have been examined. In this study, all the numerical experiments were performed on a Notebook equipped with processor Intel CoreTMi5 with 4 GB ram and 1.6 GHz CPU running U buntu Linux 12.04. The code

TestFunctions1.

$$
f(x)=\exp (-x)^{2} \sin (3 \pi x)(11)
$$

Where $0 \leq x \leq 2,3$, the radius of thespeciesr $=0.25$, in fiveattempts, the amount ofthe distribution of500vectors, result:

Table1. the real roots from the system of nonlinear equations

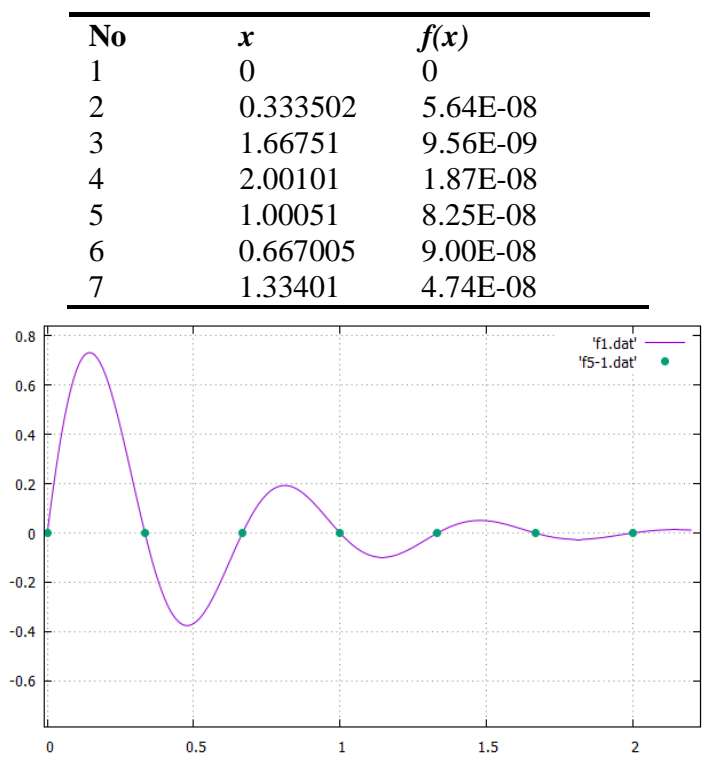

Fig4.the real roots from the system of nonlinear equations 1

Test Function 2, the system of equations and ourchosen domain are as follows: 


$$
\begin{aligned}
& f_{1}\left(x_{1}, x_{2}\right)=e^{x_{1}-x_{2}}-\sin \left(x_{1}+x_{2}=0\right. \\
& f_{2}\left(x_{1}, x_{2}\right)=x_{1}^{2} x_{2}^{1}-\cos \left(x_{1}+x_{2}\right)=0
\end{aligned}
$$

With $D=\left\{\left(x_{1}, x_{2}\right) \mid-10 \leq x_{1} \leq 10,-10 \leq x_{2} \leq 10\right\}$ the radius of thespeciesr=0.25, in fiveattempts, the amount ofthe distribution of500vectors, result:

Table2. the real roots from the system of nonlinear equations

\begin{tabular}{lll}
\hline$x 1$ & $x 2$ & $\mathrm{~F}(\mathbf{x})$ \\
0.673301 & 0.64793 & $9.41 \mathrm{E}-06$ \\
-6.14014 & -0.161162 & $3.65 \mathrm{E}-05$ \\
-0.92246 & 1.09886 & $1.07 \mathrm{E}-05$ \\
-6.45874 & 0.152943 & $1.34 \mathrm{E}-07$ \\
0.15877 & 6.03434 & 0.0147109 \\
-0.157688 & 6.46681 & 0.015597 \\
\hline
\end{tabular}

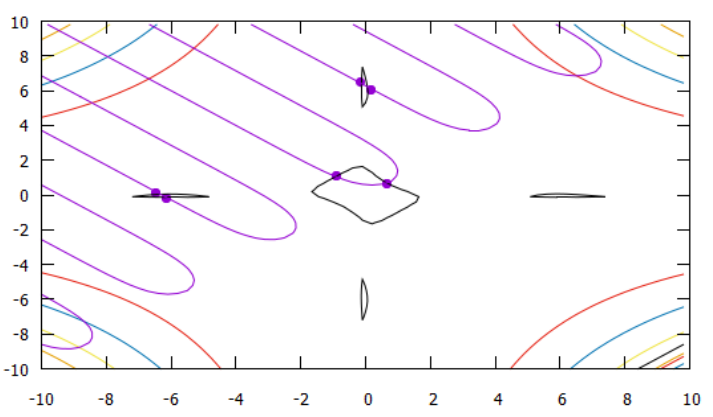

Fig 5.the real roots from the system of nonlinear equations2

\section{Conclusions}

From this research, the following conclusions can be drawn:

1. The differential evolution method utilizes vectors in a population to find the root value of a function by always having a convergent value, so that it can be used to solve root problems in non-linear functions.

2. After being tested on several benchmark functions, this method managed to get results with quite high accuracy.

3. Modification of Species-based differential evolution manages to localize all root values in the function of running a program.

4. Species-based modification in this study makes computing more efficient. 


\section{References}

[1]B. Y. Qu, P. N. Sugathan, Senior Member, IEEE, and J. J. Liang, "Differential Evolution with Neighborhood Mutation for Multimodal Optimization" in IEEE Transaction on Evolutionary Computation, 2012, Vol. 16 No. 5, pp. 601 - 613.

[2]C. Grosan and A. Abraham, "A new approach for solving Nonlinear equations systems," IEEE Trans. Systems, Man and Cybernetics, Vol.38, No.3, pp. 698-714, 2008

[3]J. Ronkkonen. "Continuous Multimodal Global Optimization With Differential Evolution-Based Methods," in Thesis for the degree of Doctor of Science (Technology),2009, Act Universitatis Lappeenrantaensis

[4]I. G. Tsoulos and A. Stavrakoudis, "On locating all roots of systems of nonlinear equations inside bounded domain using global optimization methods," Nonlinear Analysis: Real World Applications, Vol.11, pp. 2465-2471, 2010

[5]Somov, A.: Wildfire safety with wireless sensor networks. EAI Endorsed Transactions on Ambient Systems. pp. 1-11 (2011)K. V.Price, R. M. Storn, J. A. Lampinen,"Differential Evolution: A Pratical Approach to Global Optimization,” inSpringer, 2005.

[6]K. Sidarto and A. Kania, "Finding All Solutions of Systems of Nonlinear Equations Using Spiral Dynamics Inspired Optimization with Clustering," J. Adv. Comput. Intell. Intell. Inform., Vol.19, No.5, pp. 697-707, 2015

[7]Li, X.,"Efficient differential evolution using speciation for multimodal function optimization,"in Proceedings of the Conference on Genetic and Evolutionary Computation (GECCO 2005) (Washington DC, USA, 2005), pp. $873-880$.

[8]M. G. Epitropakis, V. P. Plagianakos, and M. N. Vrahatis,"Multimodal Optimization Using Niching Differential Evolution With Index-Based Neighborhoods," inWCCI 2012 IEEE World Congress on Computational Intelligence, June, 10-15, Brisbane, Australia

[9]M. Shibasaka, A. Hara, T. Ichimura, and T. Takahama. 2007. "Species-based Differential Evolution with Switching Search Strategies for Multimodal Function Optimization," inIEEE Congress on Evolutionary Computation (CEC 2007), pp. 1183 - 1190.

[10]N. Li, Y. Li, Z. Huang and Y. Wang,"Niche Differential Evolution Algorithm and Its Application in Multimodal Function Optimization,"in Advanced Materials Research Vols. 308-310 (2011) pp. 2431-2435.

[11]R. L. Burden and J. D. Faires, "Numerical Analysis," 7th ed., Brooks/Cole, 2001

[12]R. Thomsen,"Multimodal Optimization Using Crowding-Based Differential Evolution," inEvolutionary Computation. CEC2004. Congress on (Volume:2, 2004), pp 1382 - 1389.

[13]S. W. Mahfoud. "Niching Methods for Genetic Algorithms,"in Department of General Engineering, University of Illionis at Urbana-Champaign, 1995.

[14]X. Yang, "Test Problems in Optimization",in online version: http// arxiv.org/pdf/1008.0549 of the paper. 